\title{
ON TRANSPORT PROPERTIES OF ISOTROPIC QUASIPERIODIC $X Y$ SPIN CHAINS
}

\author{
ILYA KACHKOVSKIY
}

\begin{abstract}
We consider isotropic $X Y$ spin chains whose magnetic potentials are quasiperiodic and the effective one-particle Hamiltonians have absolutely continuous spectra. For a wide class of such $X Y$ spin chains, we obtain lower bounds on their Lieb-Robinson velocities in terms of group velocities of their effective Hamiltonians:$$
\mathfrak{v} \geqslant \underset{[0,1]}{\operatorname{ess} \sup } \frac{2}{\pi} \frac{d E}{d N}
$$

where $E$ is considered as a function of the integrated density of states.
\end{abstract}

\section{INTRODUCTION}

An $X Y$ spin chain is one of the most well understood models in many-body quantum physics. We will only consider isotropic $X Y$ spin chains. For an integer interval $\Lambda=[m, n] \subset \mathbb{Z}$, define the Hamiltonian as

$$
H_{\Lambda}=-\sum_{j=m}^{n}\left(\sigma_{j}^{x} \sigma_{j+1}^{x}+\sigma_{j}^{y} \sigma_{j+1}^{y}\right)-\sum_{j=m}^{n} \nu_{j} \sigma_{j}^{z},
$$

It acts in the state space

$$
\mathfrak{G}_{\Lambda}:=\otimes_{l=m}^{n} \mathbb{C}^{2}=: \otimes_{j=m}^{n} \mathfrak{G}_{j},
$$

where $\mathfrak{G}_{j}$ is the single site state space identified with $\mathbb{C}^{2}$, and the matrices $\sigma_{j}^{x, y, z}$ are the standard Pauli matrices

$$
\sigma^{x}=\left(\begin{array}{ll}
0 & 1 \\
1 & 0
\end{array}\right), \quad \sigma^{y}=\left(\begin{array}{cc}
0 & -i \\
i & 0
\end{array}\right), \quad \sigma^{z}=\left(\begin{array}{cc}
1 & 0 \\
0 & -1
\end{array}\right)
$$

acting at the respective sites, so that

$$
\sigma_{j}^{x, y, z}=I^{\otimes(j-1)} \otimes \sigma^{x, y, z} \otimes I^{\otimes(n-m-j)} .
$$

Finally $\left\{\nu_{j}\right\}_{j \in \Lambda}$ is a sequence of real numbers which is the magnetic potential.

There have been several interesting developments regarding transport properties in this model. The most well known and simple one is the Lieb-Robinson bound. If $S \subset \Lambda$, then a local observable with respect to $S$ is any operator of the form

$$
A \otimes\left(\otimes_{j \in \Lambda \backslash S} I\right),
$$

where $A$ acts in $\otimes_{j \in S} \mathfrak{G}_{j}$. We denote the algebra of all local observables on $S$ by $\mathcal{O}(S)$. Note that, formally speaking, this algebra depends on $\Lambda$, but there is a natural correspondence between $\mathcal{O}(S)$ for different $\Lambda$, so we use the same notation for them. If $A$ is an observable, then

$$
A(t):=e^{i t H_{\Lambda}} A e^{-i t H_{\Lambda}}
$$


is the Heisenberg evolution of $A$. Again, it depends on $\Lambda$. However, there are certain results (such as the following proposition) that hold for all $\Lambda$, in which case we drop the dependence on $\Lambda$ from the notation. The following is established in [15, 16].

Proposition 1.1. Suppose that $\left\{\nu_{j}\right\}_{j \in \mathbb{Z}}$ is a bounded real sequence. There exist constants $\eta, \mathfrak{v}, C$ depending only on $\sup _{j \in \mathbb{Z}}\left|\nu_{j}\right|$, such that for any finite $\Lambda \subset \mathbb{Z}$ and any two observables $A \in \mathcal{O}\left(S_{1}\right), B \in \mathcal{O}\left(S_{2}\right), S_{1}, S_{2} \subset \Lambda$, we have

$$
\|[A(t), B]\| \leqslant C\|A\|\|B\| e^{-\eta\left(\mathfrak{v} t-\operatorname{dist}\left(S_{1}, S_{2}\right)\right)} .
$$

The existence of such bound indicates that, even though the propagation speed of the Heisenberg evolution is infinite (local observables may become non-local immediately), one can still obtain an exponential bound on the tail that is sufficiently far away. In other words, the "physically effective" speed of propagation is still finite, regardless of the potential. Since there is always an upper bound, there are three interesting regimes of the transport behavior: the possible velocity can be bounded from below, can be made arbitrarily small, or can be made zero. The first case corresponds to the ballistic transport, the last case is related to localization, and the third case is an intermediate situation called anomalous transport. To describe these properties in more detail, let us note that, in a certain sense, the $X Y$ spin chain is a completely integrable system. The Jordan-Wigner transform reduces the study of this model to the study of the following effective one-particle Hamiltonian in $l^{2}(\mathbb{Z})$,

$$
\left(H_{\text {eff }} \psi\right)_{n}=\psi_{n+1}+\psi_{n-1}+\nu_{j} \psi_{j}
$$

We refer the reader to [12, 8] for the description of this transformation. We also give some additional remarks in Section 6.

The transport properties of the spin chain are related to those of the effective Hamiltonian. The zero-velocity bound is a consequence of dynamical Anderson localization, which corresponds to purely point spectrum of the effective Hamiltonian, see, for example, [12, 17, 6]. In the paper [9], a system with effective quasiperiodic Fibonacci Hamiltonian was extensively studied, and it was established that it displays anomalous transport, where one needs to replace $t$ by $t^{\alpha}$ in the exponent of the bound. This corresponds to singular continuous spectrum of $H_{\text {eff }}$. Finally, in [8], the case of periodic effective Hamiltonian was studied, and it was established that these systems admit lower bound on Lieb-Robinson velocity. This situation corresponds to absolutely continuous spectrum. The result of [8] is also proved for anisotropic $X Y$ spin chains.

While the transport properties of Schrödinger operators on $\mathbb{Z}$ are studied quite well, not all of them translate easily to the $X Y$ chain case. The reason is that the Jordan-Wigner transformation is not local, and so, the bounds on time-averaged transport exponents are not sufficient due to possible spreading of wave packets. The result of [8] for periodic potentials was obtained by showing that the lower bound on Lieb-Robinson velocity follows from existence of the following strong limit:

$$
Q=\underset{T \rightarrow+\infty}{\mathrm{S}}-\lim _{T} \frac{1}{T} \int_{0}^{T} e^{i H_{\mathrm{eff}} t} A e^{-i H_{\mathrm{eff}} t} d t
$$

where $(A \psi)_{n}=i\left(\psi_{n+1}-\psi_{n-1}\right)$. In this case, in any Lieb-Robinson bound we must have $\mathfrak{v} \geqslant 2\|Q\| 1$. Existence of this operators is the strongest form of ballistic transport: it implies

\footnotetext{
${ }^{1}$ The estimate in [8] has the form $\mathfrak{v} \geqslant\|Q\|$ because the effective Hamiltonian in their notation is $2 H_{\text {eff }}$ in ours. It is convenient for us to have the off-diagonal part of $H_{\text {eff }}$ being the usual discrete Laplacian.
} 
that all non-averaged lower transport exponents are equal to 1 . The relation between $Q$ and transport properties was first observed in [1].

In the present paper, we study the $X Y$ spin chain with quasiperiodic multi-frequency effective Hamiltonian

$$
(H(x) \psi)_{n}=\psi_{n+1}+\psi_{n-1}+v(x+n \alpha) \psi_{n} .
$$

where $\alpha$ is an irrational frequency vector and $v$ is a continuous function on a $d$-dimensional torus. We show that, under the assumption of $L^{2}$ degree 0 reducibility of the corresponding Schrödinger cocycle, there exists a non-trivial bound on possible values of $\mathfrak{v}$ in Theorem 1.1. The assumptions of the theorem hold in many cases where absolute continuity of the spectrum of $H_{\text {eff }}$ is known: for example, for analytic one-frequency potentials with Diophantine frequencies, and for analytic multi-frequency potentials at (perturbatively) small coupling. The results on concrete operators are summarized in Corollaries 3.4 and 3.6 .

Unlike the case of [8], we initially only establish existence of the phase-averaged version of $Q$. This implies that the limit (1.3) exists on a subsequence of time scales, which is still enough to obtain a velocity bound. The drawback is that it does not imply ballistic transport for $H(x)$, but implies one replaced by a phase-averaged version, see Remark 6.4.

We also give an explicit description of the lower bound in terms of the group velocity for the effective Hamiltonian:

$$
\mathfrak{v} \geqslant \underset{[0,1]}{\operatorname{ess} \sup } \frac{2}{\pi} \frac{d E}{d N},
$$

where $\frac{d E}{d N}$ is the derivative of the inverse function of the integrated density of states of $H_{\text {eff }}$. See Theorem 3.2 for precise statement.

In Section 2, we give necessary definitions in order to describe the class of quasiperiodic operators we are going to work with. In Section 3, we formulate the main results both in the language of the effective Hamiltonian and of the $X Y$ spin chain. We also describe several concrete classes of operators satisfying our assumptions. In Section 4, we summarize the properties of Aubry duality and of $L^{2}$ degree 0 reducible operators that are relevant for the proof of the main results. In Section 5, we prove Theorems 3.1 and 3.2. In Section 6 we explain the main steps of translating the language of the effective Hamiltonians to the language of $X Y$ spin chains and prove Corollary 3.3 .

\section{2. $L^{2}$-DEGREE 0 REDUCIBLE QUASIPERIODIC OPERATORS}

The main result will be formulated for an abstract class of quasiperiodic $d$-frequency operators. In order to formulate the results, we first need to introduce this class.

Let $v: \mathbb{T}^{d} \rightarrow \mathbb{R}$ be a continuous function. We will also consider $v$ as a $\mathbb{Z}^{d}$-periodic continuous function on $\mathbb{R}^{d}$. A $d$-frequency one-dimensional quasiperiodic operator family is a collection of operators of the form

$$
(H(x) \psi)_{n}=\psi_{n+1}+\psi_{n-1}+v(x+n \alpha) \psi_{n}, \quad n \in \mathbb{Z}^{d},
$$

where $\alpha=\left(\alpha_{1}, \ldots, \alpha_{d}\right) \in \mathbb{R}^{d}$ is a vector of frequencies, and $n \alpha=\left(n_{1} \alpha_{1}, \ldots, n_{d} \alpha_{d}\right)$. We assume that the set $\left\{1, \alpha_{1}, \ldots, \alpha_{d}\right\}$ is linearly independent over $\mathbb{Q}$, in which case it is an ergodic operator family with respect to the dynamics $x \mapsto x+\alpha$ on $\mathbb{T}^{d}$. The eigenvalue equation

$$
\psi_{n+1}+\psi_{n-1}+v(x+n \alpha) \psi_{n}=E \psi_{n}
$$


can be written in the following form involving transfer matrices,

$$
\left(\begin{array}{c}
\psi_{n} \\
\psi_{n-1}
\end{array}\right)=\left(\prod_{j=n-1}^{0} S_{v, E}(x+j \alpha)\right)\left(\begin{array}{c}
\psi_{0} \\
\psi_{-1}
\end{array}\right),
$$

where

$$
S_{v, E}(x)=\left(\begin{array}{cc}
E-v(x) & -1 \\
1 & 0
\end{array}\right),
$$

and the pair $\left(\alpha, S_{v, E}\right)$ is called a Schrödinger cocycle understood as a map $\left(\alpha, S_{v, E}\right): \mathbb{T}^{d} \times \mathbb{C}^{2} \rightarrow$ $\mathbb{T}^{d} \times \mathbb{C}^{2}$ given by $\left(\alpha, S_{v, E}\right):(x, w) \mapsto\left(x+\alpha, S_{v, E}(x) \cdot w\right)$. Replacing $S_{v, E}$ with $A \in \operatorname{SL}(2, \mathbb{R})$ gives a definition of an $\mathrm{SL}(2, \mathbb{R})$-cocycle.

For any Borel subset $\Delta \subset \mathbb{R}$, define density of states measure of the set $\Delta$ as

$$
N(\Delta):=\int_{\mathbb{T}^{d}}\left(\mathbb{E}_{H(x)}(\Delta) \delta_{0}, \delta_{0}\right) d x
$$

where $\mathbb{E}_{H}(\Delta)$ is the spectral projection of a self-adjoint operator $H$ in $l^{2}(\mathbb{Z})$. The integrated density of states is defined as

$$
N(E):=N((-\infty, E))=N((-\infty, E]), \quad E \in \mathbb{R} .
$$

It is known that $N(E)=1-2 \rho(E)$, where $\rho(E)$ is the fibered rotation number of the cocycle $\left(\alpha, S_{v, E}(x)\right)$.

We call an operator family (2.1) $L^{2}$-degree 0 redicible if, for almost every $E$ with respect to the density of states measure, there exists $B(\cdot, E) \in L^{2}\left(\mathbb{T}^{d} ; \mathrm{GL}(2, \mathbb{C})\right)$ such that $|\operatorname{det} B(x, E)|=1$ and

$$
B(x+\alpha, E) S_{v, E}(x) B(x, E)^{-1}=A_{\star} \quad \text { for a. e. } \quad x \in \mathbb{T}^{d},
$$

where

$$
A_{\star}=\left(\begin{array}{cc}
e^{2 \pi i \rho(E)} & 0 \\
0 & e^{-2 \pi i \rho(E)}
\end{array}\right)
$$

\section{MAIN RESUlts}

The following is the main result of the paper in terms of the effective quasiperiodic Hamiltonian.

Theorem 3.1. Let $H(x)$ be an $L^{2}$-degree 0 reducible quasiperiodic operator family. Let also $(A \psi)_{n}=i\left(\psi_{n+1}-\psi_{n-1}\right)$. There exists a full Lebesgue measure subset $\mathbb{T}_{0} \subset \mathbb{T}^{d}$ and a sequence $T_{k} \rightarrow+\infty$ as $k \rightarrow+\infty$ such that

$$
\frac{1}{T_{k}} \int_{0}^{T_{k}} e^{i H(x) t} A e^{-i H(x) t} d t \stackrel{s}{\rightarrow} Q(x), \quad \forall x \in \mathbb{T}_{0},
$$

where $Q(x)$ is a bounded operator with trivial kernel for all $x \in \mathbb{T}_{0}$, and $\|Q(x)\|$ is constant on $\mathbb{T}_{0}$. 
The integrated density of states $N(E)$ is a non-decreasing function of $E$. The inverse function $E(N)$ is defined on $[0,1]$ except maybe for a countable set of points of the form $\left\{\alpha_{1} \mathbb{Z}+\ldots+\alpha_{d} \mathbb{Z}\right\}$ which correspond to gaps in $\sigma(H)$. We can define $E(N)$ arbitrarily at these points so that the resulting function is non-decreasing on $[0,1]$ and hence differentiable almost everywhere on $[0,1]$.

Theorem 3.2. Under the assumptions of Theorem 3.1, suppose that the density of states measure of the family $H$ is absolutely continuous. Then, for almost every $x \in \mathbb{T}^{d}$,

$$
\|Q(x)\|=\frac{1}{\pi} \operatorname{ess} \sup _{[0,1]} \frac{d E}{d N} .
$$

Corollary 3.3. Suppose that an isotropic $X Y$ spin chain has effective Hamiltonian satisfying the assumptions of Theorem 3.1. Then (1.2) can only hold for all $\Lambda \subset \mathbb{Z}$ if $\mathfrak{v} \geqslant 2\|Q(x)\|$.

3.1. Concrete classes of operators. Theorems 3.1, 3.2 were formulated under some abstract assumptions. However, these assumptions hold for a wide class of operators.

Corollary 3.4. Let $H(x)$ be a one-frequency (i.e. $d=1$ ) quasiperiodic Schrödinger operator (2.1) with $v \in C^{\omega}(\mathbb{T})$, Diophantine frequency $\alpha$, and purely absolutely continuous spectrum. Then the statements of Theorems 3.1, 3.2, and Corollary 3.3 hold.

Proof. From the results of [3, 4], it follows that the operator family $H(x)$ is analytically reducible for Lebesgue almost all energies for which the Lyapunov exponent vanishes. Hence, it satisfies the assumptions of mentioned theorems.

Remark 3.5. In [5], it is shown that if $v \in C^{\omega}(\mathbb{T})$ and $\alpha$ is Diophantine, then there exists $\lambda_{0}(v)>0$ such that the spectrum of $H(x)$ with the potential $\lambda v$ will be purely absolutely continuous for $\lambda<\lambda_{0}(v)$.

Corollary 3.6. Let $H(x)$ be a multi-frequency quasiperiodic Schrödinger operator family with Diophantine frequency vector $\alpha$ and the potential $\lambda v$, where $v \in C^{\omega}\left(\mathbb{T}^{d}\right)$. There exists $\lambda_{0}(\alpha, v)>$ 0 such that, for $\lambda<\lambda_{0}(\alpha, v)$, the statements of Theorems [3.1, 3.2, and Corollary [3.3 hold.

Proof. In [10, it is shown that given $v \in C^{\omega}(T)$ and a fixed Diophantine frequency vector $\alpha$, one can find $\lambda_{0}(\alpha, v)>0$ such that $S_{\lambda v, E}$ is analytically reducible for $N$-almost every $E$ for $\lambda<\lambda_{0}(\alpha, v)$, from which all the statements follow.

\section{Properties of $L^{2}$-Degree 0 Reducible operators}

Aubry duality is a relation between spectral properties of $H(x)$ and the dual Hamiltonian $\widetilde{H}(\theta)$ which is the following operator in $l^{2}\left(\mathbb{Z}^{d}\right)$ :

$$
(\widetilde{H}(\theta) \psi)_{m}=\sum_{m^{\prime} \in \mathbb{Z}^{d}} \hat{v}_{m^{\prime}} \psi_{m-m^{\prime}}+2 \cos 2 \pi(\alpha \cdot m+\theta) \psi_{m},
$$

where

$$
v(x)=\sum_{k \in \mathbb{Z}} \hat{v}_{k} e^{2 \pi i k \cdot x} .
$$


Denote the corresponding direct integral spaces (for $H$ and $\widetilde{H}$ respectively) by

$$
\mathfrak{H}:=\int_{\mathbb{T}^{d}}^{\oplus} l^{2}(\mathbb{Z}) d x, \quad \widetilde{\mathfrak{H}}=\int_{\mathbb{T}}^{\oplus} l^{2}\left(\mathbb{Z}^{d}\right) d \theta .
$$

Consider the unitary operator $\mathcal{U}: \mathfrak{H} \rightarrow \widetilde{\mathfrak{H}}$ defined on vector functions $\Psi=\Psi(x, n)$ as

$$
(\mathcal{U} \Psi)(\theta, m)=\hat{\Psi}(m, x+\alpha \cdot m)
$$

where $\hat{\Psi}$ denotes the Fourier transform over $x \in \mathbb{T}^{d} \rightarrow m \in \mathbb{Z}^{d}$ combined with the inverse Fourier transform $n \in \mathbb{Z} \rightarrow \theta \in \mathbb{T}$. Let also

$$
\mathcal{H}:=\int_{\mathbb{T}^{d}}^{\oplus} H(x) d x, \quad \widetilde{\mathcal{H}}:=\int_{\mathbb{T}}^{\oplus} \widetilde{H}(\theta) d \theta .
$$

Aubry duality can be formulated as the following equality of direct integrals.

$$
\mathcal{U} \mathcal{H} \mathcal{U}^{-1}=\tilde{\mathcal{H}}
$$

It is well known (see, for example, [11]) that the spectra of $H(x)$ and $\widetilde{H}(\theta)$ coincide for all $x, \theta$. We denote then both by $\Sigma$. Moreover, the IDS of the families $H$ and $\widetilde{H}$ also coincide. We will use the following properties of the fibered rotation number and $L^{2}$-degree 0 reducibility, see [13] and references therein for more detail.

(1) The rotation number is a continuous non-increasing function of $E$. It is locally constant on $\mathbb{R} \backslash \Sigma$, where $\Sigma=\sigma(H(x)$ ) (this set does not depend on $x$ ), and its values on $\mathbb{R} \backslash \Sigma$ are $\frac{1}{2} \mathbb{Z}$-linear combinations of $\alpha_{1}, \ldots, \alpha_{d}$. It maps $\Sigma$ onto $[0,1 / 2]$.

(2) Suppose that (2.3) holds for some $A_{\star}$ for $N$-almost every $E$ with $B$ continuous in $x$. Then, $N$-almost every $E$, it also holds with $A_{\star}$ given by (2.4). In other words, a continuously reducible quasiperiodic operator family is automatically degree 0 reducible.

(3) For almost every $\theta \in[0,1 / 2]$, there exists a unique $E \in \mathbb{R}$ such that $\rho(E)=\theta$. Denote this $E$ by $E(\theta)$. For almost every $\theta \in[0,1 / 2]$, the cocycle $S_{v, E(\theta)}$ satisfies (2.3). By $f(x, \theta)$, denote the matrix element $\left(B^{-1}(x, E(\theta))\right)_{11}$. The function $f$ can be chosen to be $L^{2}$-normalized in $x$ and measurable in $\theta$. We assume that from now on.

(4) Extend $f(x, \theta)$ to $\theta \in[-1 / 2,0]$ by $f(x,-\theta):=\overline{f(x, \theta)}$, and then extend it with period 1 to all $\theta \in \mathbb{R}$. Then, for almost every $\theta$, the dual Hamiltonian $\widetilde{H}(\theta)$ has purely point spectrum with eigenvalues $E(\theta-k \cdot \alpha), k \in \mathbb{Z}^{d}$, and eigenvectors $u(\theta, k)_{m}=$ $\hat{f}(m+k, \theta-k \alpha)$, where $\hat{f}$ is the Fourier transform of $f$ over $x$. We use the convention that the lower index $m \in \mathbb{Z}^{d}$ of the vector enumerates its components, $k \in \mathbb{Z}^{d}$ enumerates different eigenvectors of the same operator, and $\theta$ is the ergodic parameter enumerating the operators $\widetilde{H}(\theta)$.

(5) For almost every $\theta$, the function $d(\theta)=e^{2 \pi i \theta} f(x, \theta) \overline{f(x-\alpha, \theta)}-e^{-2 \pi i \theta} \overline{f(x, \theta)} f(x-\alpha, \theta)$ is well defined (i.e. does not depend on $x$ almost surely) and non-zero. For these $\theta$, one can choose $B(x)^{-1}$ of the form

$$
\frac{1}{d(\theta)^{1 / 2}}\left(\begin{array}{cc}
f(x, \theta) & \overline{f(x, \theta)} \\
e^{-2 \pi i \theta} f(x-\alpha, \theta) & e^{2 \pi i \theta} \overline{f(x-\alpha, \theta)}
\end{array}\right) .
$$

In the sequel, we will denote the matrix $B(x)$ obtained for $E=E(\theta)$ by $B(x, \theta)$. 


\section{Proofs of Theorems 3.1 and 3.2}

We first need a few auxiliary results. Suppose that $\mathfrak{H}=\int_{\mathbb{T}^{d}}^{\oplus} l^{2}(\mathbb{Z}) d x$. We will consider bounded decomposable operators of the form $\mathcal{H}=\int_{\mathbb{T}^{d}}^{\oplus} H(x) d x$, where $H(\cdot)$ is an a. e. uniformly bounded measurable family of operators.

Proposition 5.1. Suppose that $\mathcal{H}_{n}=\int_{\mathbb{T}^{d}}^{\oplus} H_{n}(x) d x$ is a sequence of bounded decomposable operators. Then

(1) If $\left\|H_{n}(x)\right\| \leqslant C$ for a. e. $x \in \mathbb{T}^{d}$ and all $n \in \mathbb{N}$, and $H_{n}(x) \stackrel{s}{\rightarrow} H(x)$ for $a$. e. $x \in \mathbb{T}^{d}$, then $\mathcal{H}_{n} \stackrel{s}{\rightarrow} \int_{\mathbb{T}^{d}}^{\oplus} H(x) d x$.

(2) If $\mathcal{H}_{n} \stackrel{s}{\rightarrow} \mathcal{H}$, where $\mathcal{H}$ is a bounded operator on $\int_{\mathbb{T}^{d}}^{\oplus} l^{2}(\mathbb{Z}) d x$, then $\mathcal{H}$ is a bounded decomposable operator, and there exists a subset $\mathbb{T}_{0} \subset \mathbb{T}^{d}$ of full Lebesgue measure and a subsequence $\left\{n_{k}\right\}$ such that $H_{n_{k}}(x) \stackrel{s}{\rightarrow} H(x)$ for $x \in \mathbb{T}_{0}$.

(3) $\mathcal{H}$ has trivial kernel if and only if $H(x)$ has trivial kernel for a. e. $x \in \mathbb{T}^{d}$.

Proof. The first claim follows from the dominated convergence theorem applied to the integral

$$
\int_{T}\left\|H_{n}(x) f(x)-H(x) f(x)\right\| d x
$$

where $f: \mathbb{T}^{d} \rightarrow l^{2}(\mathbb{Z})$ is an element of $\int_{\mathbb{T}^{d}}^{\oplus} l^{2}(\mathbb{Z}) d x$. To prove the second claim, denote by $e_{k}(\cdot) \in \int_{\mathbb{T}}^{\oplus} l^{2}(\mathbb{Z}) d x$ the constant vector function $x \mapsto \delta_{k}$. We have $\mathcal{H}_{n} e_{k}(\cdot) \stackrel{s}{\rightarrow} \mathcal{H} e_{k}(\cdot)$ in $\int_{\mathbb{T}}^{\oplus} l^{2}(\mathbb{Z}) d x$. Hence, $\int_{\mathbb{T}^{d}}\left\|H_{n}(x) e_{k}-H(x) e_{k}\right\|^{2} d x \rightarrow 0$. Since $L^{2}$-convergence implies convergence in measure, we get that there exists a subsequence such that $H_{n_{l}}(x) e_{k} \rightarrow H(x) e_{k}$ for almost every $x$. Applying Cantor diagonal procedure, we can ensure that there exists a set of $x$ of full measure and a subsequence that converges on all basis vectors $e_{k}$. Since this sequence is also uniformly bounded, by Banach-Steinhaus theorem, there will be strong operator convergence on that subsequence. Third claim is well known.

Lemma 5.2. Let $A$ be a bounded operator and $H$ be a bounded self-adjoint operator with purely point spectrum. Let $\left\{\lambda_{l}\right\}$ be the distinct eigenvalues of $H$, and $P_{l}=\mathbb{E}_{H}\left\{\lambda_{l}\right\}$ be the projection onto the corresponding eigenspace. Then

$$
\underset{T \rightarrow+\infty}{\mathrm{S}-\lim _{\mathrm{T}}} \frac{1}{T} \int_{0}^{T} e^{i H t} A e^{-i H t} d t=\sum_{l} P_{l} A P_{l} .
$$

The right hand side can be considered as the "diagonal part" of $A$ with respect to the eigenspaces of $J$.

Proof. The left hand side is uniformly bounded in T. Due to Banach-Steinhaus theorem, it is sufficient to check the convergence on the eigenvectors of $H$. Let $\psi_{l, j}$ be the eigenvectors of $H$, $J \psi_{l, j}=\lambda_{l} \psi_{l, j}$, where $j$ enumerates different eigenvectors from the same eigenspace. We have

$$
\begin{gathered}
\frac{1}{T} \int_{0}^{t} e^{i H t} A e^{-i H t} d t \psi_{l, j}=\sum_{l^{\prime}, k} \frac{1}{T} \int_{0}^{T} e^{i\left(\lambda_{l^{\prime}}-\lambda_{l}\right) t} d t\left(A \psi_{l, j}, \psi_{l^{\prime}, k}\right) \psi_{l^{\prime}, k}= \\
=P_{l} A P_{l} \psi_{l, j}+\sum_{l^{\prime} \neq l} \sum_{k} \frac{e^{i\left(\lambda_{l^{\prime}}-\lambda_{l}\right) T}-1}{T i\left(\lambda_{l^{\prime}}-\lambda_{l}\right)}\left(A \psi_{l, j}, \psi_{l^{\prime}, k}\right) \psi_{l^{\prime}, k}=
\end{gathered}
$$




$$
=P_{l} A P_{l} \psi_{l, j}+\sum_{l^{\prime} \neq l} e^{i\left(\lambda_{l^{\prime}}-\lambda_{l}\right) T / 2} \frac{\sin \left(\left(\lambda_{l^{\prime}}-\lambda_{l}\right) T / 2\right)}{\left(\lambda_{l^{\prime}}-\lambda_{l}\right) T / 2} P_{l^{\prime}} A \psi_{l, j}
$$

To show that the last sum converges to 0 , note that the terms are mutually orthogonal, and $\sum_{l^{\prime} \neq l}\left\|P_{l^{\prime}} A \psi_{l, j}\right\|^{2} \leqslant\left\|A \psi_{l, j}\right\|^{2}$. Hence, given $\varepsilon>0$, there exists $N$ such that the sum over $l^{\prime}>N$ is norm bounded by $\varepsilon$ for all $T>0$. The sum over $l^{\prime}<N$ has finite number of terms each of which goes to 0 as $T \rightarrow+\infty$.

Lemma 5.3. Under the assumptions of Theorem 3.1, let $\widetilde{H}(\theta)$ be the dual operator family. Let also $(\widetilde{A}(\theta) \psi)_{n}=2 \sin (2 \pi(n \alpha+\theta)) \psi_{n}$. Then, for almost every $\theta \in \mathbb{T}$, there exists a strong limit

$$
\widetilde{Q}(\theta)=\mathrm{s}_{T \rightarrow \infty} \lim \frac{1}{T} \int_{0}^{T} e^{i \widetilde{H}(\theta) t} \widetilde{A}(\theta) e^{-i \widetilde{H}(\theta) t} d t
$$

$\widetilde{Q}(\theta)$ has trivial kernel for almost every $\theta \in \mathbb{T}$.

Proof. Suppose that $\theta$ is chosen to fulfill Properties $3-5$ from the previous subsection. Then the operator $\widetilde{H}(\theta)$ has purely point spectrum, and the convergence is established by Lemma 5.2. We only need to show that $\operatorname{ker} \widetilde{Q}(\theta)=\{0\}$. The operator $\widetilde{Q}(\theta)$ is diagonal in the basis $u(\theta, k)$ of eigenvectors of $\widetilde{H}(\theta)$. Let us compute its diagonal entries, that is,

$$
\widetilde{Q}(\theta) u(\theta, k)=\left\{\sum_{m \in \mathbb{Z}^{d}} 2 \sin (2 \pi(m \cdot \alpha+\theta))\left|u(\theta, k)_{m}\right|^{2}\right\} u(\theta, k), \quad k \in \mathbb{Z}^{d},
$$

where $u(\theta, k)_{m}=\hat{f}(m+k, \theta-k \alpha)$ are normalized eigenvectors of $\widetilde{H}(\theta)$. We need to show that none of the diagonal entries (i.e. sums in curly brackets) are zero. Let us first take $k=0$, and denote $u(\theta)_{m}=u(\theta, 0)_{m}$. Then

$$
\begin{gathered}
\sum_{m} 2 \sin (2 \pi(m \cdot \alpha+\theta))\left|u(\theta)_{m}\right|^{2}=2 \operatorname{Im} \sum_{m} e^{2 \pi i(m \cdot \alpha+\theta)} \hat{f}(m, \theta) \overline{\hat{f}(m, \theta)} \\
=2 \operatorname{Im} \int_{\mathbb{T}^{d}} e^{2 \pi i \theta} f(x+\alpha, \theta) \overline{f(x, \theta)} d x \\
=\int_{\mathbb{T}^{d}}\left\{e^{2 \pi i \theta} f(x+\alpha, \theta) \overline{f(x, \theta)}-e^{-2 \pi i \theta} \overline{f(x+\alpha, \theta)} f(x, \theta)\right\} d x=d(\theta) \neq 0
\end{gathered}
$$

for almost every $\theta$ by Property 5 . The case $k \neq 0$ is obtained by replacing $\theta$ with $\theta+k \cdot \alpha$. The set of $\theta$ such that the last quantity is non-zero for all $k \in \mathbb{Z}$ has full measure as a countable intersection of full measure sets.

Remark 5.4. From the proof, it is easy to see that $\|\widetilde{Q}(\theta)\|=\|\widetilde{Q}(\theta+k \cdot \alpha)\|$, and that it is a measurable function of $\theta$. Hence, $\|\widetilde{Q}(\theta)\|$ is almost surely constant in $\theta$.

Proof of Theorem 3.1. Let $\mathcal{U}$ be the duality operator (4.2). We have

$$
\mathcal{U}\left(\int_{\mathbb{T}^{d}}^{\oplus} e^{i H(x) t} A e^{-i H(x) t} d x\right) \mathcal{U}^{-1}=\int_{\mathbb{T}}^{\oplus} e^{i \widetilde{H}(\theta) t} \widetilde{A}(\theta) e^{-i \widetilde{H}(\theta) t} d \theta
$$


Since the operators $\widetilde{H}(\theta)$ have purely point spectra for almost all $\theta$, Lemma 5.3 and Proposition 5.1 imply that the Cesaro averages of the right hand side converge to a bounded operator with non-zero kernel which we will denote by $\widetilde{\mathcal{Q}}$. Hence,

$$
\frac{1}{T} \int_{0}^{T}\left\{\int_{\mathbb{T}^{d}}^{\oplus} e^{i H(x) t} A e^{-i H(x) t} d x\right\} d t \stackrel{s}{\rightarrow} \mathcal{Q}=\mathcal{U}^{-1} \widetilde{\mathcal{Q}} \mathcal{U}
$$

By Proposition 5.1, $\mathcal{Q}$ is decomposable, and so $\mathcal{Q}=\int_{\mathbb{T}}^{\oplus} Q(x) d x$, where $Q(x)$ has trivial kernel for almost every $x$. Existence of a subsequence follows from Proposition [5.1, and the fact that $\|Q(x)\|$ is almost surely constant follows from the fact that $Q(x+\alpha)$ is unitary equivalent to $Q(x)$.

Proof of Theorem 3.2. The argument is fairly standard, and was used (with (5.4) obtained), for example, in [4] for the case of analytically reducible cocycles. For almost every $\theta$, we can consider

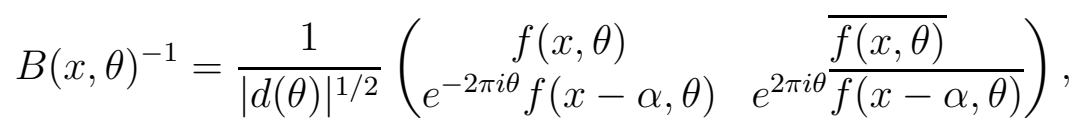

where $d(\theta)=e^{2 \pi i \theta} f(x, \theta) \overline{f(x-\alpha, \theta)}-e^{-2 \pi i \theta} \overline{f(x, \theta)} f(x-\alpha, \theta)$ does not depend on $x$. We have

$$
B(x+\alpha, \theta) S_{v, E}(x) B(x, \theta)^{-1}=\left(\begin{array}{cc}
e^{2 \pi i \theta} & 0 \\
0 & e^{-2 \pi i \theta}
\end{array}\right),
$$

and $\operatorname{det} B(x, \theta)= \pm i$. Take $J:=\mp \frac{1}{\sqrt{2}}\left(\begin{array}{cc}1 & 1 \\ -i & i\end{array}\right)$. Define the new matrix $\widetilde{B}(x, \theta):=J B(x, \theta) \in$ $\operatorname{SL}(2, \mathbb{R})$. We have

$$
\widetilde{B}(x+\alpha, \theta) S_{v, E}(x) \widetilde{B}(x, \theta)^{-1}=\left(\begin{array}{cc}
\cos \theta & -\sin \theta \\
\sin \theta & \cos \theta
\end{array}\right)=: R_{\theta} .
$$

From the proof of Lemma 5.3 and (5.2), it follows that, for almost all $\theta$,

$$
\|\widetilde{Q}(\theta)\|=\sup _{k \in \mathbb{Z}}|d(\theta+k \alpha)|=\sup _{k \in \mathbb{Z}} \frac{4}{\int_{\mathbb{T}^{d}}\left\|B(x, \theta+k \alpha)^{-1}\right\|_{\mathrm{HS}}^{2} d x}=\sup _{k \in \mathbb{Z}} \frac{4}{\int_{\mathbb{T}^{d}}\|\widetilde{B}(x, \theta+k \alpha)\|_{\mathrm{HS}}^{2} d x},
$$

where HS denotes the Hilbert-Schmidt norm; note that $B$ and $B^{-1}$ have the same norms.

We now need to recall some results from Kotani theory, see, for example, [7]. The formula

$$
\frac{d N}{d E}=\frac{1}{2 \pi} \int_{\mathbb{T}^{d}} \frac{1}{\operatorname{Im} m(E, x)} d x
$$

is valid for almost every $E$ for which $\gamma(E)=0$. Here $m$ is the $m$-function, or a measurable invariant section of the hyperbolic action of $S_{v, E}(x)$ with the properties

$$
\operatorname{Im} m(E, x)>0, \quad m(E, x+\alpha)=S_{v, E}(x) \cdot m(E, x),
$$

where "." denotes the hyperbolic action of an $\mathrm{SL}(2, \mathbb{R})$-matrix on the upper half plane, that is,

$$
\left(\begin{array}{ll}
a & b \\
c & d
\end{array}\right) \cdot z=\frac{a z+b}{c z+d} .
$$


Note that $L^{2}$-reducibility of $S_{v, E}$ implies that $\gamma(E)=0$. Since we assume that $N$ is absolutely continuous, Kotani's formula is valid for almost every $E$ with respect to $N$. If $C(x+\alpha) S_{v, E}(x) C(x)^{-1} \in \mathrm{SO}(2, \mathbb{R})$ with some $C \in \mathrm{SL}(2, \mathbb{R})$, then one can check that

$$
\|C(x)\|_{\mathrm{HS}}^{2}=\frac{1}{\operatorname{Im} C(x)^{-1} \cdot i}+\frac{1}{\operatorname{Im} C(x+\alpha)^{-1} \cdot i} .
$$

Kotani's theory also implies that, for Lebesgue almost every $E$ with $\gamma(E)=0$ (which, in our notation, would also be for almost every $\theta)$, there exists $C(\cdot, \theta) \in L^{2}\left(\mathbb{T}^{d}, \operatorname{SL}(2, \mathbb{R})\right)$ such that

$$
C(x+\alpha, \theta) S_{v, E}(x) C(x, \theta)^{-1} \in \mathrm{SO}(2, \mathbb{R}), \quad C(x, \theta)^{-1} \cdot i=m(x, E) .
$$

We claim that, even though our matrix $\widetilde{B}$ may be different from $C$, we also have $\widetilde{B}(x, \theta)^{-1} \cdot i=$ $m(x, E)$. Indeed, take $u(x):=\widetilde{B}(x, \theta) C(x, \theta)^{-1} \cdot i$. We have $R_{\theta} \cdot u(x)=u(x+\alpha)$. The set of $x \in \mathbb{T}^{d}$ such that $u(x)=i$ has either zero or full measure (since $R_{\theta}$ preserves $i$ ). Assume that it has zero measure and take $w(x)=\frac{u(x)-i}{u(x)+i}$. A simple computation shows that $w(x)$ is a measurable unitary function satisfying $e^{2 \pi i \theta} w(x)=w(x+\alpha)$. This is only possible if $\theta \in \alpha_{1} \mathbb{Z}+\ldots \alpha_{d} \mathbb{Z}+\mathbb{Z}$. If we exclude these $\theta$, we can assume that $u(x)=i$ for almost every $x$, and hence $\widetilde{B}(x, \theta)^{-1} \cdot i=m(x, E)$. This implies that

$$
\frac{d N}{d E}=\frac{1}{4 \pi} \int_{\mathbb{T}^{d}}\|\widetilde{B}(x, \theta)\|_{\mathrm{HS}}^{2} d x .
$$

From (5.3), we obtain that

$$
\|\widetilde{Q}(\theta)\|=\left.\sup _{k \in \mathbb{Z}} \frac{1}{\pi}\left(\frac{d N}{d E}\right)^{-1}\right|_{E=E(\theta+k \alpha)}=\underset{[0,1]}{\operatorname{ess} \sup } \frac{1}{\pi} \frac{d E}{d N}
$$

for almost every $\theta$.

\section{Proof of Corollary 3.3}

Corollary 3.3 follows from the following result.

Theorem 6.1. Suppose that an isotropic XY spin chain satisfies the assumptions of Theorem 1.1, and, for some real sequence $T_{k} \rightarrow+\infty$,

$$
\frac{1}{T_{k}} \int_{0}^{T_{k}} e^{i H_{\mathrm{eff}} t} A e^{-i H_{\mathrm{eff}} t} d t \stackrel{s}{\rightarrow} Q .
$$

Then, (1.2) can only hold for all $\Lambda \subset \mathbb{Z}$ if $\mathfrak{v} \geqslant 2\|Q\|$.

This result was essentially proved in [8] with two minor differences. The first one is that we only assume that the limit exists on a subsequence. This difference is really minor and it can be easily traced that the proof remains the same. The other difference is that we only consider isotropic spin chains, in which case the effective Hamiltonian decouples. This simplifies some computations, and we choose to include some proofs in order to keep the text more selfcontained. 
Proposition 6.2. [8] Under the assumptions of Theorem [6.4, for any $\varepsilon>0$ there exists $k(\varepsilon) \in$ $\mathbb{N}$ and constants $C(\varepsilon), L(\varepsilon)$ such that for any $k \geqslant K(\varepsilon)$ one can find $l, r \in \mathbb{Z}$ with $|l| \leqslant K(\varepsilon)$ and

$$
(\|Q\|-\varepsilon) T_{k} \leqslant|r| \leqslant(\|Q\|+\varepsilon) T_{k}
$$

such that

$$
\left|\left(\delta_{r}, e^{-i T_{k} H_{\mathrm{eff}}} \delta_{l}\right)\right|^{2} \geqslant \frac{C}{T_{k}}
$$

Proof. Let $v=\|Q\|$. Without loss of generality, one can assume that $\chi_{[v-\varepsilon / 2, v]}(Q) \neq 0$. There exists $l \in \mathbb{Z}, l \leqslant K(\varepsilon)$, such that $\chi_{[v-\varepsilon / 2, v]}(Q) \delta_{l} \neq 0$. Let us now relate the operator $Q$ with transport properties. Denote by $X$ the position operator in $l^{2}(\mathbb{Z})$,

$$
(X u)_{n}=n u_{n}
$$

defined on the natural domain. The operator $Q$ is related with the Heisenberg evolution of $X$. We have $\operatorname{Dom} X(T)=\operatorname{Dom} X$, and

$$
X(T) u=X u+\int_{0}^{T} A(t) u d t=X u+\int_{0}^{T} e^{i H t} A e^{-i H t} d t, \quad u \in \operatorname{Dom} X .
$$

where

$$
A=\overline{i[X, H]}, \quad(A \psi)_{n}=-i \psi_{n-1}+i \psi_{n+1} .
$$

We have $\frac{1}{T_{k}} X\left(T_{k}\right) u \rightarrow Q u$ for any $u \in \operatorname{Dom} X$ as $k \rightarrow \infty$. This implies that the sequence $\frac{1}{T_{k}} X\left(T_{k}\right)$ converges to $Q$ in the strong resolvent sense, see Theorem VIII.25 from [18]. Let $\varphi$ be a continuous non-negative function equal to 1 on $[v-\varepsilon / 2, v]$ and vanishing outside $[v-\varepsilon, v+\varepsilon]$. Due to Theorem VIII.20 from [18], we have

$$
\varphi\left(\frac{1}{T_{k}} X\left(T_{k}\right)\right) u \rightarrow \varphi(Q) u, \quad \forall u \in \operatorname{Dom} X
$$

and so

$$
\left\|\chi_{\left[T_{k}(v-\varepsilon), T_{k}(v+\varepsilon)\right]}\left(X\left(T_{k}\right)\right) \delta_{l}\right\| \geqslant\left\|\varphi\left(X\left(T_{k}\right) / T_{k}\right) \delta_{l}\right\| \geqslant C
$$

for sufficiently large $k$. The indicator function in the left hand side is simply the sum of projections onto $\delta_{r}$ with $r \in\left[T_{k}(v-\varepsilon), T_{k}(v+\varepsilon)\right]$. Hence,

$$
\sum_{r \in\left[T_{k}(v-\varepsilon), T_{k}(v+\varepsilon)\right]}\left|\left(\delta_{r}, e^{-i T_{k} H} \delta_{l}\right)\right|^{2} \geqslant C,
$$

and so, for some $r \in\left[T_{k}(v-\varepsilon), T_{k}(v+\varepsilon)\right]$, we have

$$
\left|\left(\delta_{r}, e^{-i T_{k} H} \delta_{l}\right)\right|^{2} \geqslant \frac{C}{2 \varepsilon T_{k}+1} \geqslant \frac{C_{1}}{T_{k}} \text {. }
$$

Let us now consider the $X Y$ spin chain on $\Lambda=[m, n] \cap \mathbb{Z}$, and construct the following observables

$$
\begin{gathered}
a_{j}^{*}:=\frac{1}{2}\left(\sigma_{j}^{x}+i \sigma_{j}^{y}\right), \quad a_{j}:=\frac{1}{2}\left(\sigma_{j}^{x}-i \sigma_{j}^{y}\right), \\
c_{m}:=a_{m}, \quad c_{m+j}:=\sigma_{m}^{z} \sigma_{m+1}^{z} \ldots \sigma_{m+j-1}^{z} a_{m+j}, \quad 1 \leqslant j \leqslant n-m .
\end{gathered}
$$

Let also $H_{\mathrm{eff}}^{\Lambda}:=\left.H_{\mathrm{eff}}\right|_{[m, n]}$ be the restriction of the original operator onto $[m, n] \cap \mathbb{Z}$. 
Proposition 6.3. Let $m \leqslant l \leqslant r \leqslant n$. Then

$$
\left\|\left[c_{l}(t), a_{r}^{*}\right]\right\| \geqslant\left|\left(e^{-2 i t H_{\text {eff }}^{\Lambda}} \delta_{l}, \delta_{r}\right)\right| .
$$

Proof. It was shown in [12] that, if $C^{\Lambda}=\left(c_{m}, \ldots, c_{n}\right)^{T}$, then

$$
C^{\Lambda}(t)=e^{-2 i t H_{\text {eff }}} C^{\Lambda} \text {. }
$$

Consider the following special state

$$
u_{\Lambda}=\bigotimes_{j=m}^{n}\left(\begin{array}{l}
1 \\
0
\end{array}\right) \in \mathfrak{G}_{\Lambda} .
$$

A simple application of commutation relations between $c_{l}$ and $a_{r}^{*}$ shows that

$$
\left\|\left[c_{l}(t), a_{r}^{*}\right]\right\| \geqslant\left|\left[c_{l}(t), a_{r}^{*}\right] u_{\Lambda}\right|=\left|\left(e^{-2 i t H_{\mathrm{eff}}^{\Lambda}} \delta_{l}, \delta_{r}\right)\right|
$$

which completes the proof.

Proof of Theorem 6.1. Suppose that Lieb-Robinson bound holds with the velocity $\mathfrak{v}$. Fix some $\varepsilon>0$ and obtain $l, r$ from Proposition 6.2. Due to Proposition 6.3 with $t=T_{k} / 2$ and because of Lieb-Robinson bound, we have

$$
\left|\left(e^{-i T_{k} H_{\text {eff }}^{\Lambda}} \delta_{l}, \delta_{r}\right)\right| \leqslant\left\|\left[c_{l}(t), a_{r}^{*}\right]\right\| \leqslant C e^{-\eta\left(|r-l|-\mathfrak{v} T_{k} / 2\right)} .
$$

This inequality must hold for all $\Lambda \subset \mathbb{Z}$, and hence (after taking strong limit) we have the following for $H_{\text {eff }}$ if $k \geqslant K(\varepsilon)$ :

$$
\frac{C}{\sqrt{T_{k}}} \leqslant\left|\left(e^{-i T_{k} H_{\text {eff }}} \delta_{l}, \delta_{r}\right)\right| \leqslant C_{1} e^{-\eta\left(|r-l|-\mathfrak{v} T_{k} / 2\right)} \leqslant C_{1} e^{-\eta\left((\|Q\|-\varepsilon) T_{k}-L(\varepsilon)-1-\mathfrak{v} T_{k} / 2\right)} .
$$

This inequality must hold for arbitrarily large $k$, which is only possible if $\mathfrak{v} \geqslant 2(\|Q\|-\varepsilon)$. Since $\varepsilon$ is arbitrary, this completes the proof.

Remark 6.4. The (non-averaged) lower transport exponent is defined as

$$
\beta_{\psi}^{-}(p)=\liminf _{T \rightarrow+\infty} \frac{\log (X(T) \psi, \psi)}{p \log t} .
$$

One can show that, under the assumptions of Theorem, we have

$$
\liminf _{k \rightarrow \infty} \frac{1}{T_{k}^{p}}\left(\left|X\left(T_{k}\right)\right| \psi, \psi\right) \geqslant\left(|Q|^{p} \psi, \psi\right)>0,
$$

for any $\psi \neq 0$ with finite support. If the limit (6.1) existed as $T \rightarrow \infty$ (not on a subsequence), one would be able to show that $\beta_{\psi}^{-}(p)=1$ for any $p>0$. In the case of quasiperiodic operators as in Theorem 3.1, one can show that

$$
\liminf _{T \rightarrow \infty} \frac{1}{T} \int_{\mathbb{T}^{d}}\left(|X(T, x)|^{p} \psi, \psi\right) d x \geqslant \int_{\mathbb{T}^{d}}\left(|Q(x)|^{p} \psi, \psi\right)>0,
$$

where $X(T, x)=e^{i T H(x)} X e^{-i T H(x)}$. Since $x$ is the ergodic parameter, (6.2) can be understood as presence of ballistic transport in expectation. 


\section{Acknowledgements}

I would like to thank Svetlana Jitomirskaya for drawing my attention to the problem. I would also like to thank her, as well as Vojkan Jaksic, Milivoje Lukic and Gunter Stölz, for valuable discussions. I am grateful to Isaac Newton Institute for Mathematical Sciences, Cambridge, for support and hospitality during the programme Periodic and Ergodic Spectral Problems where part of this paper was completed. The research was supported by AMS-Simons Travel Grant, 2014-2016, and partially by NSF DMS-1401204.

\section{REFERENCES}

[1] Asch J., Knauf A., Motion in periodic potentials, Nonlinearity 11 (1998), 175 - 200.

[2] Aubry S. André G., Analyticity breaking and Anderson localization in incommensurate lattices, Group theoretical methods in physics (Proc. Eighth Internat. Colloq., Kiryat Anavim, 1979), pp. 133 - 164, Ann. Israel Phys. Soc., 3, Hilger, Bristol, 1980.

[3] Avila A., Krikorian R., Reducibility or nonuniform hyperbolicity for quasiperiodic Schrödinger cocycles, Annals of Mathematics 164 (2006), 911 - 940.

[4] Avila A., Fayad B., Krikorian R., A KAM scheme for $\mathrm{SL}(2, \mathbb{R})$ cocycles with Liouvillean frequencies. Geom. Funct. Anal. 21 (2011), $1001-1019$.

[5] Bourgain J., Jitomirskaya S., Absolutely continuous spectrum for 1D quasiperiodic operators, Invent. Math. 148 (2002), $453-463$.

[6] Chapman J., Stolz G., Localization for random block operators related to the XY spin chain, Annales Henri Poincaré 16 (2015), no. 2, $405-435$.

[7] Damanik D., Lyapunov exponents and spectral analysis of ergodic Schrödinger operators: A survey of Kotani theory and its applications, Spectral theory and mathematical physics: a Festschrift in honor of Barry Simon's 60th birthday, 539 - 563, Proc. Sympos. Pure Math., 76, Part 2, Amer. Math. Soc., Providence, RI, 2007.

[8] Damanik D., Lukic M., Yessen W., Quantum dynamics of periodic and limit-periodic Jacobi and block Jacobi matrices with application to some quantum many body problems, http://arxiv.org/abs/1407.5067v2.

[9] Damanik D., Lemm M., Lukic M., Yessen W., On anomalous Lieb-Robinson bounds for the Fibonacci XY chain, http://arxiv.org/abs/1407.4924v1.

[10] Eliasson L., Floquet solutions for the 1-dimensional quasi-periodic Schrödinger equation, Comm. Math. Phys. 146 (1992), no. 3, $447-482$.

[11] Gordon A., Jitomirskaya S., Last Y., Simon B., Duality and singular continuous spectrum in the almost Mathieu equation, Acta Mathematica 178 (1997), 169 - 183.

[12] Hamza E., Sims R., Stolz G., Dynamical Localization in Disordered Quantum Spin Systems, Comm. Math. Phys 315 (2012), no. 1, $215-239$.

[13] Jitomirskaya S., Kachkovskiy I., $L^{2}$-reducibility and localization for quasiperiodic operators, preprint

[14] Last Y., Quantum dynamics and decompositions of singular continuous spectra, Journal of Functional Analysis 42 (1996), 406 - 445.

[15] Lieb E., Robinson D., The finite group velocity of quantum spin systems, Comm. Math. Phys. 28 (1972), $251-257$.

[16] Nachtergaele B., Sims R., Locality estimates for quantum spin systems, V. Siboravičius (ed.), New trends of mathematical physics. Selected contributions of the XVth international congress on mathematical physics. Springer (2009), $591-614$.

[17] Sims R., Stolz G., Many-body localization: concepts and simple models, preprint, http://arxiv.org/abs/1312.0577v1

[18] Reed M., Simon B., Methods of modern mathematical physics I: functional analysis, Academic Press, 1972. 